\title{
INDEPENDENCE RESULTS ON THE GLOBAL STRUCTURE OF THE TURING DEGREES
}

\author{
BY
}

\author{
MARCIA J. GROSZEK AND THEODORE A. SLAMAN ${ }^{1}$
}

"For nothing worthy proving can be proven, Nor yet disproven." Tennyson

\begin{abstract}
From CON(ZFC) we obtain:
1. $\operatorname{CON}\left(\mathrm{ZFC}+2^{\omega}\right.$ is arbitrarily large + there is a locally finite upper semilattice of size $\omega_{2}$ which cannot be embedded into the Turing degrees as an upper semilattice).

2. $\mathrm{CON}\left(\mathrm{ZFC}+2^{\omega}\right.$ is arbitrarily large + there is a maximal independent set of Turing degrees of size $\omega_{1}$ ).
\end{abstract}

Introduction. Let $\mathscr{D}$ denote the set of Turing degrees ordered under the usual Turing reducibility, viewed as a partial order $\langle\mathscr{D}, \leqslant\rangle$ or an upper semilattice $\langle\mathscr{D}, \leqslant, \vee\rangle$ depending on context. A partial order $\langle A, \leqslant\rangle$ [upper semilattice $\langle A, \leqslant, \vee\rangle]$ is embeddable into $\mathscr{D}$ (denoted $A \rightarrow \mathscr{D}$ ) if there is an embedding $f$ : $A \rightarrow \mathscr{D}$ so that $a \leqslant b$ if and only if $f(a) \leqslant f(b)$ [and $f(a) \vee f(b)=f(a \vee b)$ ].

The structure of $\mathscr{D}$ was first investigted in the germinal paper of Kleene and Post [2] where $A \rightarrow \mathscr{D}$ for any countable partial order $A$ was shown. Sacks [9] proved that $A \rightarrow \mathscr{D}$ for any partial order $A$ which is locally finite (any point of $A$ has only finitely many predecessors) and of size at most $2^{\omega}$, or locally countable and of size at most $\omega_{1}$.

Say $X \subseteq \mathscr{D}$ is an independent set of Turing degrees if, whenever $x_{0}, x_{1}, \ldots, x_{n} \in X$ and $x_{0} \leqslant x_{1} \vee x_{2} \vee \cdots \vee x_{n}$, there is an $i$ between 1 and $n$ so that $x_{0}=x_{i} ; X$ is maximal if no proper extension of $X$ is independent. Sacks showed (also in [9]) that no countable set of Turing degrees is maximal independent and that there is an independent set of Turing degrees of size continuum. Sacks conjectured that in fact any maximal independent set of Turing degrees must have size continuum. In [11], S. Simpson pointed out that $\mathrm{MA}(\kappa)$ implies that every maximal independent set has size greater than $\kappa$, but suggested that the existence of a maximal independent set of size less than continuum might be independent of ZFC. Theorem 2 confirms Simpson's conjecture.

Received by the editors March 30, 1982.

1980 Mathematics Subject Classification. Primary 03D30.

Key words and phrases. Turing degrees, forcing, uncountable embeddings, independent sets.

${ }^{1}$ The second author was supported by an NSF Postdoctoral Fellowship MSC 81-14165. The authors wish to thank Gerald Sacks for bringing these matters to our attention.

(C)1983 American Mathematical Society 0002-9947/82/0000-0685/\$03.50 
This result casts some light (or shadow, if you will) on the following conjecture, also made in [9] and still unsettled: "If $A$ is a partial order which is locally countable and of size at most continuum then $A \rightarrow \mathscr{D}$ ". The existence of a small maximal independent set means that a simple extension of embeddings method, such as works for $A$ of size $\omega_{1}$, will not suffice.

The embedding of upper semilattices into $\mathscr{D}$ has been studied in the context of this question: "Which upper semilattices can be represented as initial segments of QD?"-all the finite ones, Sacks first conjectured in [9]. The following (listed historically, without regard for redundancy) are now known to be embeddable in $\mathscr{D}$ as initial segments: every countable distributive upper semilattice with least element (Lachlan, 1968 [4]), every finite lattice (Lerman, 1971 [6]), every countable upper semilattice with least element (Lachlan and Lebeuf, 1976 [5]), $\omega_{1}$ and many other upper semilattices of size $\omega_{1}$ (Rubin, $1979[7,8]$ ).

Theorem 1 shows that $\omega_{1}$ is in some sense an upper bound; there is a model where $2^{\omega}$ is large yet there is a locally finite upper semilattice of size $\omega_{2}$ which is not embeddable in $\mathscr{D}$. (By Sacks's result in [9], being locally finite, it can be embedded as a partial order.)

Notation. A real $x$ will be viewed as a function from $\omega$ into $2(=\{0,1\}) .\{e\}$ denotes the $e$ th partial recursive function and $\{e\}^{x}$ denotes the $e$ th partial recursive function in the real $x$. If $Y$ is a set then $\overline{\bar{Y}}$ is its cardinality.

We will be working with finite sequences of integers, so let Seq be the set of finite sequences of 0 's and 1's (Seq $=\{s \mid \exists n<\omega[s: n \rightarrow 2]\}$ ). Seq is naturally ordered by inclusion. If $s$ is a sequence then $\ln (s)$ is its length (= domain $(s))$. Finally, if $s$ is a finite sequence then $s^{\wedge} i$ is defined by

$$
s^{\wedge} i(m)= \begin{cases}s(m) & \text { if } m<\ln (s), \\ i & \text { if } m=\ln (s) .\end{cases}
$$

The notation used to describe the forcing constructions is as standard as possible. $M$ is used to denote the ground model. $\mathbf{P}$ is the forcing partial order and $\mathcal{G}$ is the generic subset of P. $M[\mathcal{G}]$ is the result of extending $M$ generically by $\mathcal{G}$. If $x$ is an element of $M[\mathcal{G}], \stackrel{\circ}{x}$ will denote a term in the forcing language which names $x$. If $x$ is in $M, \hat{x}$ denotes its canonical name in the forcing language. On the other hand, if $x$ is a term in the forcing language, $(x)^{N}$ is its realization in the model $N$. Unless these notations are necessary for clarity or emphasis, we write simply $x$.

$\mathbb{I}_{\mathbf{P}}$ denotes the forcing relation for the partial order $\mathbf{P}$. When the intended partial order is clear $\mathbb{t}$ is used. Our convention is that $p \leqslant q$ means the forcing condition $p$ is stronger (says more) than the condition $q$.

THEOREM 1. Let $M \vDash$ "ZFC $+2^{\omega}=\omega_{1}+\kappa \in \mathrm{CARD}+\mathrm{cf}(\kappa) \geqslant \omega_{1}$ ”. There is a cardinal-preserving generic extension $M[\mathcal{G}]$ of $M$ so that: $M[\mathcal{G}] \vDash " 2^{\omega}=\kappa \&$ there is an upper semilattice $U$ so that:

(i) $\overline{\bar{U}}=\omega_{2}$;

(ii) $U$ is locally finite (any element of $U$ has only fintely many predecessors);

(iii) $U \nrightarrow \mathscr{D} "$. 
The model $M[\mathcal{G}]$ is obtained by adding $\kappa$ Cohen reals to $M$ (see Cohen [3]) via a finite support product forcing. $U$ is constructed in $M$ so that there are $\omega_{2}$ elements in $U$, each of which has a distinct uncountable behavior with respect to join in $U$. Analysis of the forcing shows that there are only $\omega_{1}$ possible ways that a Turing degree in $M[\mathcal{G}$ ] can behave with respect to the ground model.

We have chosen Cohen forcing for its simplicity of definition and to make it easy for the reader to verify the standard (and hence unproven) forcing lemmata (Lemmas 1 and 2). The heart of the proof, however, is a simple counting argument, which depends on the homogeneous character of product forcing. Similar arguments apply to other methods of expanding the continuum via product forcing.

Definition. The Cohen forcing partial order. Let $\kappa$ be a cardinal of uncountable cofinality. Define $\mathbf{P}_{\boldsymbol{\kappa}}$ and $\leqslant$ by

$$
\begin{gathered}
\mathbf{P}_{\kappa}=\{p: \kappa \rightarrow \operatorname{Seq} \mid\{\alpha<\kappa \mid p(\alpha) \neq \varnothing\} \text { is finite }\} ; \\
p \leqslant q \text { iff }(\forall \alpha<\kappa)[p(\alpha) \supseteq q(\alpha)] .
\end{gathered}
$$

LEMMA 1 [3]. $\mathbf{P}_{\kappa}$ has the countable chain condition (ccc).

Corollary. If $M[\mathcal{G}]$ is a $\mathbf{P}_{\kappa}$-generic extension of $M$ and $M \vDash \mathrm{CH}$ then $M$ [G] has the same cardinals as $M$ and $M[\mathcal{G}] \vDash " 2^{\omega}=\kappa "$.

LEMMA 2. If $X$ is a set of reals in $M[\mathcal{G}]$ and $\overline{\bar{X}}=\omega_{1}$ in $M[\mathcal{G}]$, then there is an intermediate model $N$ ( $M \subseteq N \subseteq M[\mathcal{G}])$ so that:

(i) $X \in N$;

(ii) $N$ is a generic extension of $M$ by $\mathbf{P}_{\omega_{1}}$;

(iii) if $\kappa>\omega_{1}$, then $M[\mathcal{G}]$ is a generic extension of $N$ by $\mathbf{P}_{\kappa}$.

Lemma 2 is proven using an automorphism of $\mathbf{P}_{\kappa}$, from the fact that $\mathbf{P}_{\kappa}$ has the ccc.

Definition. The upper semilattice. Let $\left\langle X_{\beta} \mid \beta<\omega_{2}\right\rangle$ be $\omega_{2}$-many distinct subsets of $\omega_{1}$ in $M$. Let $U$ be the upper semilattice generated by

generators: $\{a\} \cup\left\{b_{\alpha} \mid \alpha<\omega_{1}\right\} \cup\left\{c_{\beta} \mid \beta<\omega_{2}\right\}$;

relations: $a<b_{\alpha} \vee c_{\beta}$ iff $\alpha \in X_{\beta}$.

Proposition. If $M$ and $\kappa$ are as in the hypothesis of Theorem 1 , and $\mathcal{G}$ is $\mathbf{P}_{\kappa}$-generic over $M$, then

$$
M[\mathcal{G}] \vDash \text { “ }\langle U, \leqslant, \vee\rangle \nrightarrow\langle\mathscr{D}, \leqslant, \vee\rangle " .
$$

Proof. Assume there is an embedding $f: U \rightarrow \mathscr{D}$ in $M[\mathcal{G}]$. By Lemma 2 we may first pass to an intermediate extension and assume that $f(a)$ and $\left\langle f\left(b_{\alpha}\right) \mid \alpha<\omega_{1}\right\rangle$ are elements of $M$. Again invoking Lemma 2, each $f\left(c_{\beta}\right)$ is in some extension of $M$ by $\mathbf{P}_{\omega_{1}}$. Since (Lemma 1) when forcing over $M$, $\mathbb{t}_{\mathbf{P}_{\omega_{1}}}$ " $2^{\omega}=\omega_{1}$ ", there is in $M$ a collection of terms $\left\langle t_{\gamma} \mid \gamma<\omega_{1}\right\rangle$ so that any real in a $\mathbf{P}_{\omega_{1}}$-generic extension of $M$ realizes one of the $t_{\gamma}$. In particular, $\omega_{2}$ of the $f\left(c_{\beta}\right)$ contain reals realizing some fixed $t_{\gamma_{0}}$ in some extension of $M$ by $\mathbf{P}_{\omega_{1}}$. But then, for each such $\beta$, there must be a $p \in \mathbf{P}_{\omega_{1}}$ so that for each $\alpha<\omega_{1}$,

$$
p \Vdash \text { " } \hat{f}(a)<\hat{f}\left(b_{\alpha}\right) \vee \operatorname{deg}\left(t_{\gamma_{0}}\right) \quad \text { iff } \alpha \in \hat{X}_{\beta} " .
$$

The $X_{\beta}$ are all distinct, yet $\overline{\overline{\mathbf{P}}}_{\omega_{1}}=\omega_{1}$, a contradiction. This proves Theorem 1 . 
THEOREM 2. Let $M \vDash$ " $\mathrm{ZFC}+2^{\omega}=\omega_{1}+\kappa \in \mathrm{CARD}+\mathrm{cf}(\kappa) \geqslant \omega_{1}$ ". There is a cardinal-preserving generic extension of $M, M[\mathcal{G}] \Vdash{ }^{\prime}$ " ${ }^{\omega}=\kappa \&$ there is a maximal independent set of Turing degrees of size $\omega_{1}$ ".

In outline, the proof is as follows. First add $\omega_{1}$ mutually generic Sacks reals (see Sacks [10]) to $M$ via a countable support product forcing. Their Turing degrees form a (maximal) independent set $X$. Furthermore (Lemma 6 ) any degree in $M$ is below uncountably many degrees in $X$.

Then add to this intermediate model $\kappa$ mutually generic Sacks reals. The resulting model satisfies $2^{\omega}=\kappa$ and has the same cardinals as $M$. Also (Lemma 7 ) any new real computes any of the intermediate generic reals when joined with some ground model real. This shows that $X$ remains maximal in the final model.

The operative fact here is that the branching conditions and fusion sequences of Sacks forcing allow complicated reals to be coded into a condition, whence they can be recursively recovered by the generic. Thus a Sacks real introduces via join many new relations of relative computability between ground model reals. Lemmata 6 and 7 are manifestations of this phenomenon.

With the exception of these two, the assertions made during the course of the definitions and proof are standard facts about Sacks forcing, product forcing, and iterated forcing. The truth of the two Fusion Lemmata and the Minimality Lemma is easily checked.

Most of the complexity occurs in the definition of a "P-fusion sequence for $x$ ". To minimize this, some modifications have been made in the basic definitions: The definition of a "fusion sequence for $P$ " is slightly weaker than (but essentially equivalent to) the usual definition. The explicit definition of the partial order $\mathbf{P}$ is not that of a two-step iteration of product forcings; however, in this case, the two can easily be seen to be equivalent.

Definitions. Sacks forcing. $T$ is a perfect tree if $T$ is a nonempty subset of Seq, $T$ is downward closed, and every element of $T$ has incomparable extensions in $T$. A node $t$ splits in $T$ if $t^{\wedge} 0 \in T$ and $t^{\wedge} 1 \in T$. Stem $(T)$ is the least node which splits in $T$. The nth splitting level of $T$ is denoted $L_{n}(T)$ and defined by

$$
L_{n}(T)=\{t \mid t \text { splits in } T \& \overline{\overline{\{s \subsetneq t \mid s \text { splits in } T\}}}=n\} .
$$

$T_{s}$ is $T$ above $s$ and defined by

$$
T_{s}=\{t \in T \mid t \subseteq s \text { or } s \subseteq t\} .
$$

The Sacks forcing partial order $P$ is the set of perfect trees. $P$ is ordered by reverse inclusion ( $p \leqslant q$ if $p \subseteq q$ ).

A generic set $\mathcal{G} \subseteq P$ is identified with a $P$-generic real $G=\cap\{T \mid T \in \mathcal{G}\}$.

Say $S \leqslant_{m} T$ iff $S \leqslant T$ and $L_{m}(S)=L_{m}(T) .\left\langle T_{n} \mid n<\omega\right\rangle$ is a fusion sequence for $P$ iff for all $n, T_{n} \supseteq T_{n+1}$ and

$$
(\exists f: \omega \rightarrow \omega)(\forall m)(\forall n \geqslant f(m))\left[T_{n+1} \leqslant_{m} T_{n}\right] .
$$

LEMMA 3 (FUsion). If $\left\langle T_{n}\right| n\langle\omega\rangle$ is a fusion sequence for $P$ then its fusion $T_{\omega}=\bigcap_{n<\omega} T_{n}$ is an element of $P$. 
Definition. If $T \Vdash$ " $x$ : $\omega \rightarrow 2 \& x \notin M$ " we can define a $P$-fusion sequence for $x$ below $T,\left\langle T_{n} \mid n<\omega\right\rangle$, so that $T_{0}=T$;

$$
\begin{aligned}
(\forall n)\left[T_{n+1} \leqslant\right. & T_{n} \&\left(\forall s \in L_{n}\left(T_{n}\right)\right)(\exists k<\omega)(\exists i<2) \\
& {\left.\left[\left(T_{n+1}\right)_{s^{\wedge} 0} \Vdash x(k)=i \&\left(T_{n+1}\right)_{s^{\wedge} 1} \Vdash x(k) \neq i\right]\right] . }
\end{aligned}
$$

$\left\langle T_{n}\right| n\langle\omega\rangle$ is defined by induction. Given $T_{n}$, let $L_{n}\left(T_{n}\right)=\left\{s_{0}, \ldots, s_{N}\right\}$. Since $T_{0} \Vdash x \notin M$, for each $s_{j}$ there is a $k_{j}$ so that $\left(T_{n}\right)_{s_{j} 0}$ does not decide $x\left(k_{j}\right)$. Let $T_{j}^{\prime} \leqslant\left(T_{n}\right)_{s_{j}, 1}$ so that $T_{j}^{\prime} \Vdash x\left(k_{j}\right)=i_{j}$. Let $T_{j}^{\prime \prime} \leqslant\left(T_{n}\right)_{s_{j} \hat{0}}$ so that $T_{j}^{\prime \prime} \Vdash x\left(k_{j}\right) \neq i_{j}$. Define $T_{n+1}=\cup_{0 \leqslant j \leqslant N}\left(T_{j}^{\prime} \cup T_{j}^{\prime \prime}\right)$.

Lemma 4 (Minimality [10]). If $T \Vdash$ “ $x: \omega \rightarrow 2 \& x \notin M$ ” and $\left\langle T_{n} \mid n<\omega\right\rangle$ is a $P$-fusion sequence for $x$ below $T$, then its fusion $T_{\omega} \leqslant T$ and

$$
T_{\omega} \Vdash \text { “ } G=\bigcup\left\{s \in T_{\omega} \mid(\forall k<\omega)(\forall i<2)\left[\left(\left(T_{\omega}\right)_{s} \Vdash x(k)=i\right) \Rightarrow x(k)=i\right]\right\} \text { ". }
$$

The $P$-fusion sequence together with the associated facts about the forcing relation on $x$ form a reduction procedure which can be used to compute the generic from $x$.

Corollary. If $G$ is P-generic over $M$ then

$$
M[G] \vDash(\forall x: \omega \rightarrow 2)\left[x \notin M \Rightarrow\left(\exists z \in\left({ }^{\omega} 2\right)^{M}\right)\left[G \leqslant_{T}\langle x, z\rangle\right]\right] .
$$

Definitions. The forcing partial order $\mathbf{P}$. $\mathbf{P}$ is a two-step iterated forcing, of which the first step is a countable support size $\omega_{1}$ product of Sacks forcing (see Baumgartner and Laver [1]), and the second a countable support size $\kappa$ product of Sacks forcing. Let $R$ be defined by

$$
R=\left\{r \mid \operatorname{dom}(r)=\omega_{1} \&\left(\forall \alpha<\omega_{1}\right)[r(\alpha) \in P] \&\{\alpha \mid r(\alpha) \neq \text { Seq }\} \text { is countable }\right\} .
$$

$R$ is ordered by $r \leqslant \bar{r}$ if and only if for all $\alpha<\omega_{1}, r(\alpha) \subseteq \bar{r}(\alpha)$. A generic $\mathcal{G} \subseteq R$ defines a sequence $G$ of mutually Sacks generic reals. (For $\alpha<\omega_{1}, G(\alpha)=$ $\cap\{p(\alpha) \mid p \in \mathcal{G}\})$.

Define $\mathbf{P}$ by

$$
\begin{aligned}
& \mathbf{P}=\left\{p \mid \operatorname{dom}(p)=\kappa \& p \uparrow \omega_{1} \in R\right. \\
& \quad \& p \uparrow \omega_{1} \Vdash_{R} “\left(\forall \alpha \in \kappa-\omega_{1}\right)[p(\alpha) \in P] ” \\
& \left.\quad \&\left\{\alpha \in \kappa-\omega_{1} \mid p \uparrow \omega_{1} \nVdash_{R} p(\alpha)=\text { Seq }\right\} \text { is countable }\right\} .
\end{aligned}
$$

$\mathbf{P}$ is ordered by

$$
p \leqslant \bar{p} \text { iff } p \nmid \omega_{1} \leqslant \bar{p} \uparrow \omega_{1} \& p \uparrow \omega_{1} \mathbb{\Vdash}_{R} \text { “ }\left(\forall \alpha \in \kappa-\omega_{1}\right)[p(\alpha) \subseteq \bar{p}(\alpha)] ” .
$$

If $\mathcal{G}$ is a generic subset of $\mathbf{P}$ then $\mathcal{G}$ defines a generic sequence of reals $G$ by:

$G(\alpha)=\bigcap\{p(\alpha) \mid p \in \mathcal{G}\}$ if $\alpha<\omega_{1}$

$G \uparrow \omega_{1}$ is $R$-generic over $M$ so let $M_{1}=M\left[G \uparrow \omega_{1}\right]$;

$G(\alpha)=\bigcap\left\{(p(\alpha))^{M_{1}} \mid p \in \mathcal{G}\right\}$ for $\alpha \in \kappa-\omega_{1}$.

Let $M_{2}$ be $M[G]$.

The support of a condition $p$ is

$\operatorname{supp}(p)=\left\{\alpha \mid \alpha<\omega_{1} \& p(\alpha) \neq \operatorname{Seq}\right.$ or $\left.\alpha \in \kappa-\omega_{1} \& p \uparrow \omega_{1} \nVdash{ }_{R} “ p(\alpha)=\operatorname{Seq} "\right\}$. 
Definition. $\left\langle p_{n} \mid n<\omega\right\rangle$ is a fusion sequence for $\mathbf{P}$ if

(i) $\left(\forall \alpha<\omega_{1}\right)\left[\left\langle p_{n}(\alpha) \mid n<\omega\right\rangle\right.$ is a fusion sequence for $\left.P\right]$ (hence $p_{\omega} \mid \omega_{1} \in R$ when $\left.p_{\omega}(\alpha)=\bigcap_{n<\omega} p_{n}(\alpha)\right)$, and

(ii) $\left(\forall \alpha \in \kappa-\omega_{1}\right)\left[p_{\omega} \mid \omega_{1} \Vdash_{R} "\left\langle p_{n}(\alpha) \mid n<\omega\right\rangle\right.$ is a fusion sequence for $P$ "].

The fusion of $\left\langle p_{n} \mid n \in \omega\right\rangle$ is $p_{\omega}$ and is defined as

$$
p_{\omega}(\alpha)= \begin{cases}\bigcap_{n<\omega} p_{n}(\alpha) & \text { if } \alpha<\omega_{1}, \\ \bigcap_{n<\omega}\left(p_{n}(\alpha)\right)^{M_{1}} & \text { if } \alpha \in \kappa-\omega_{1} .\end{cases}
$$

LeMma 5. If $\left\langle p_{n} \mid n<\omega\right\rangle$ is a fusion sequence for $\mathbf{P}$ then its fusion $p_{\omega}$ is a condition in $\mathbf{P}$.

Definition. If $p \Vdash$ “ $x: \omega \rightarrow 2 \& x \in M_{2}-M_{1}$ ”, define a P-fusion sequence for $x$ below $p$ by

$$
\left\langle p_{s} \leqslant p \mid s \in \operatorname{Seq}\right\rangle,\left\langle\sigma_{s} \in \operatorname{Seq} \mid s \in \operatorname{Seq}\right\rangle,\left\langle\alpha_{n}<\kappa \mid n<\omega\right\rangle
$$

satisfying:

(1) $p_{s} \Vdash$ “ $x \uparrow \ln \left(\sigma_{s}\right)=\sigma_{s}$ " and if $s \subset t$ then $\sigma_{s} \subset \sigma_{t}$ and $p_{t} \leqslant p_{s}$.

(2) $(\forall n)\left(\forall s \in{ }^{n} 2\right)\left(\forall t \in{ }^{n} 2\right)$

$$
\left.\begin{array}{c}
\left(\forall \alpha<\omega_{1}\right)\left[\begin{array}{c}
(\exists m<n)\left[\alpha=\alpha_{m} \& s(m) \neq t(m)\right] \\
\Rightarrow p_{s}(\alpha) \text { and } p_{t}(\alpha) \text { have incomparable stems }
\end{array}\right) \\
\&\left(\begin{array}{l}
\sim(\exists m<n)\left[\alpha=\alpha_{m} \& s(m) \neq t(m)\right] \\
\Rightarrow p_{s}(\alpha)=p_{t}(\alpha)
\end{array}\right) \\
\&\left(p_{s} \uparrow \omega_{1}=p_{t} \uparrow \omega_{1}\right) \Rightarrow p_{s} \uparrow \omega_{1} \Vdash
\end{array}\right]
$$

Condition (2) says that $p_{n}=W_{\ln (s)=n} p_{s}$ is well defined by

$$
p_{n}(\alpha)= \begin{cases}\bigcup_{\substack{\ln (s)=n \\ \ln (s)=n, p_{s} \backslash \omega_{1} \in G \backslash \omega_{1}}} p_{s}(\alpha) & \text { if } \alpha<\omega_{1},\end{cases}
$$

(3) $(\forall \alpha)\left[(\exists s)\left[\alpha \in \operatorname{supp}\left(p_{s}\right)\right] \Rightarrow\left\{n \mid \alpha=\alpha_{n}\right\}\right.$ is unbounded in $\left.\omega\right]$.

Condition (3) says that $\left\langle p_{n}\right| n\langle\omega\rangle$ is a fusion sequence for $\mathbf{P}$, with fusion

$$
p_{\omega}=\bigwedge_{n<\omega} \underset{\ln (s)=n}{W} p_{s}
$$

Thus, if $\alpha$ is in $\operatorname{supp}\left(p_{\omega}\right) \cap \omega_{1}$ then

$$
p_{\omega} \Vdash " G(\alpha)=\bigcap_{p_{s} \in G} p_{s}(\alpha) " .
$$


If $\alpha$ is in $\operatorname{supp}\left(p_{\omega}\right) \cap\left(\kappa-\omega_{1}\right)$ then

$$
p_{\omega} \Vdash " G(\alpha)=\bigcap_{p_{s} \in G}\left(p_{s}(\alpha)\right)^{M_{1}} " .
$$

(4) If $\ln (s)=\ln (t)=n$ and $\left(\exists \alpha<\omega_{1}\right)(\exists m<n)\left[\alpha=\alpha_{m} \& s(m) \neq t(m)\right]$, then $\sigma_{s}$ and $\sigma_{t}$ are incomparable.

Condition (4) implies that

$$
p_{\omega} \Vdash \text { " }(\forall s)\left[p_{s} \uparrow \omega_{1} \in G \uparrow \omega_{1} \text { iff } \sigma_{s}=x \uparrow \ln \left(\sigma_{s}\right)\right] ” .
$$

Construction of a fusion sequence for $x$. Let $p \Vdash$ " $x: \omega \rightarrow 2 \& x \in M_{2}-M_{1}$ ". Assume that condition (3) is satisfied by a suitable diagonalization strategy. Let $p_{\langle\rangle}=p, \sigma_{\langle\rangle}=\langle\rangle$. Inductively, suppose $\left\langle p_{s} \mid \ln (s)=n\right\rangle$ and $\left\langle\sigma_{s} \mid \ln (s)=n\right\rangle$ have been constructed, and $\alpha_{n}$ is given. For $\ln (s)=n$ let $\sigma_{s^{\prime} i}^{0}=\sigma_{s}$,

$$
p_{s i}^{0} \hat{i}_{i}(\alpha)= \begin{cases}p_{s}(\alpha), & \alpha \neq \alpha_{n}, \\ \left(p_{s}(\alpha)\right)_{\operatorname{stem}\left(p_{s}(\alpha)\right)_{i},}, & \alpha=\alpha_{n} .\end{cases}
$$

If $\alpha_{n} \in \kappa-\omega_{1}$ then $p_{s^{\wedge} i}=p_{s^{\wedge} i}^{0}$ and $\sigma_{\hat{s}{ }_{i}}=\sigma_{s^{\prime} i}^{0}$ will suffice as condition (4) only requires $p_{s}$ and $p_{t}$ to force different facts about $x$ when $p_{s} \uparrow \omega_{1} \neq p_{t} \uparrow \omega_{1}$ and the splitting necessary for condition (2) has been accomplished.

Otherwise, let $\left\{\left\langle t_{l}, \bar{t}_{l}\right\rangle \mid 1 \leqslant l \leqslant k\right\}$ order all pairs $\langle t, \bar{t}\rangle$ so that

$$
\sim(\exists m<n)\left[\alpha_{m}<\omega_{1} \& t(m) \neq \bar{t}(m)\right]
$$

but $t(n) \neq \bar{t}(n)$. Inductively (on $j$ ) construct $\left\langle p_{s}^{j}, \sigma_{s}^{j} \mid \ln (s)=n+1\right\rangle$ so that this sequence satisfies conditions (1) and (2) as well as condition (4) for pairs $\left\langle t_{l}, \bar{t}_{l}\right\rangle$ when $l<j$.

The resulting $\left\langle p_{s}^{k+1}, \sigma_{s}^{k+1} \mid \ln (s)=n+1\right\rangle$ will be as desired.

Suitable $p_{s}^{0}$ and $\sigma_{s}^{0}$ have already been described, so suppose $\left\langle p_{s}^{j}, \sigma_{s}^{j} \mid \ln (s)=n+1\right\rangle$ given and describe $p_{s}^{j+1}$ and $\sigma_{s}^{j+1}$.

Once $p_{s}^{j+1}$ and $\sigma_{s}^{j+1}$ have been defined for $s=t_{j}$ and $s=\bar{t}_{j}$, then define for the remaining $s, \sigma_{s}^{j+1}=\sigma_{s}^{j}$ and

$$
p_{s}^{j+1}(\alpha)=\left\{\begin{array}{ll}
p_{t_{j}}^{j+1}(\alpha) & \text { if } p_{s}^{j}(\alpha)=p_{t_{j}}^{j}(\alpha) \\
p_{t_{j}}^{j+1}(\alpha) & \text { if } p_{s}^{j}(\alpha)=p_{t_{j}}^{j}(\alpha) \\
p_{s}^{j}(\alpha) & \text { otherwise } \\
p_{t_{j}}^{j+1}(\alpha) & \text { if } p_{s}^{j+1} \uparrow \omega_{1}=p_{t_{j}}^{j+1} \uparrow \omega_{1} \text { and } \\
p_{s}^{j+1} \uparrow \omega_{1} \Vdash “ p_{s}^{j}(\alpha)=p_{t_{j}}^{j}(\alpha) ” \\
p_{t_{j}}^{j+1}(\alpha) & \text { if } p_{s}^{j+1} \uparrow \omega_{1}=p_{t_{j}}^{j+1} \uparrow \omega_{1} \text { and } \\
p_{s}^{j+1} \uparrow \omega_{1} \Vdash “ p_{s}^{j}(\alpha)=p_{t_{j}}^{j}(\alpha) ” \\
p_{s}^{j}(\alpha) & \text { otherwise }
\end{array}\right\}, \quad \alpha \geqslant \omega_{1} \text {. }
$$

This is to say that $p_{s}^{j}(\alpha)$ is extended to agree with $p_{t_{j}}^{j+1}(\alpha)$ and/or $p_{t_{j}}^{j+1}(\alpha)$ when necessary to satisfy condition (2). 
It remains only to define $p_{t_{j}}^{j+1}, p_{t_{j}}^{j+1}, \sigma_{t_{j}}^{j+1}$ and $\sigma_{t_{j}}^{j+1}$. Since $p \Vdash x \notin M_{1}$,

$$
p_{t_{j}}^{j} \Vdash_{R}(\exists N)\left[p_{t_{j}}^{j} \uparrow\left(\kappa-\omega_{1}\right) \text { does not decide } x(N) \text { in } M_{1}\right] \text {. }
$$

That is,

$$
\begin{gathered}
\left(\exists q \leqslant p_{t_{j}}^{j}\right)(\exists N)\left[q \uparrow\left(\kappa-\omega_{1}\right)=p_{t_{j}}^{j} \uparrow\left(\kappa-\omega_{1}\right) \&(\forall \bar{q} \leqslant q)\right. \\
{\left[\bar{q} \uparrow\left(\kappa-\omega_{1}\right)=q \uparrow\left(\kappa-\omega_{1}\right)\right.} \\
\Rightarrow(\forall i) \sim[\bar{q} \Vdash x(N)=i]]] .
\end{gathered}
$$

Fix $q$, whose existence is stated above, and define $\bar{p} \leqslant p_{t_{j}}^{j}$ by

$$
\bar{p}(\alpha)= \begin{cases}q(\alpha) & \text { if } \alpha<\omega_{1} \text { and } \alpha \neq \alpha_{n}, \\ p_{t_{j}}^{j}(\alpha) & \text { if } \alpha \geqslant \omega_{1} \text { or } \alpha=\alpha_{n} .\end{cases}
$$

$\bar{p}$ extends $p_{t_{j}}^{j}$ since we have been inductively satisfying conditions (1) and (2). That is, either we already have a difference forced about $x$ by $p_{t_{j}}^{j}$ and $p_{t_{j}}^{j}$ (contrary to assumption), or $p_{t_{j}}^{j}$ and $p_{t_{j}}^{j}$ are identical below $\omega_{1}$ except for $\alpha_{n}$. Choose $\overline{\bar{p}} \leqslant \bar{p}$ and $\sigma_{t_{j}}^{j+1} \in \in^{N+1} 2$ so that

$$
\overline{\bar{p}} \Vdash “ s \uparrow(N+1)=\sigma_{\bar{t}_{j}}^{j+1} " .
$$

Define $\overline{\bar{p}} \leqslant q$ by

$$
\overline{\bar{p}}(\alpha)= \begin{cases}\overline{\bar{p}}(\alpha) & \text { if } \alpha<\omega_{1} \text { and } \alpha \neq \alpha_{n}, \\ q(\alpha) & \text { if } \alpha \geqslant \omega_{1} \text { or } \alpha=\alpha_{n} .\end{cases}
$$

Since $\overline{\bar{p}}$ is different from $q$ only below $\omega_{1}$ and $\overline{\bar{p}}$ extends $q$, it cannot decide $x(N)$. This was the feature which defined $q$ and allows condition (4) to be satisfied. Choose $p_{t_{j}}^{j+1} \leqslant \overline{\bar{p}}$ and $\sigma_{t_{j}}^{j+1}$ so that $\sigma_{t_{j}}^{j+1}(N) \neq \sigma_{t_{j}}^{j+1}(N)$ and $p_{t_{j}}^{j+1} \Vdash$ " $x \uparrow N+1=\sigma_{t_{j}}^{j+1}$ ". Define $p_{t_{j}}^{j+1} \leqslant \overline{\bar{p}}^{j}$ by

$$
p_{t_{j}}^{j+1}(\alpha)= \begin{cases}p_{t_{j}}^{j+1}(\alpha) & \text { if } \alpha<\omega_{1} \text { and } \alpha \neq \alpha_{n}, \\ \overline{\bar{p}}(\alpha) & \text { if } \alpha \geqslant \omega_{1} \text { or } \alpha=\alpha_{n} .\end{cases}
$$

This completes the construction.

Lemma 6. If $p \in \mathbf{P}, z: \omega \rightarrow 2$ is in $M$, and $\alpha \notin \operatorname{supp}(p)$, then there is a condition $\bar{p} \leqslant p$ so that $\bar{p} \Vdash$ “ $\operatorname{deg}(z) \leqslant \operatorname{deg}(G(\alpha))$ ” (where $\operatorname{deg}$ denotes Turing degree).

Corollary. If $z: \omega \rightarrow 2$ is in $M$ then, in $M_{1},\left\{\alpha<\omega_{1} \mid \operatorname{deg}(z) \leqslant \operatorname{deg}(G(\alpha))\right\}$ is unbounded in $\omega_{1}$.

Proof of Lemma 6. Define a tree $T_{z}$ in $P$ by

$$
T_{z}=\{s \in \operatorname{Seq} \mid(\forall n)[(2 n \leqslant \ln (s) \& s(2 n)=i) \Rightarrow z(n)=i]\} .
$$

Then $T_{z} \Vdash_{P}$ " $z(n)=i$ iff $G(2 n)=i$ ", hence $T_{z} \Vdash_{P}$ “ $\operatorname{deg}(z) \leqslant \operatorname{deg}(G)$ ". Define $\bar{p}$ by

$$
\bar{p}(\bar{\alpha})= \begin{cases}p(\bar{\alpha}) & \text { if } \bar{\alpha} \neq \alpha, \\ T_{z} & \text { if } \bar{\alpha}=\alpha .\end{cases}
$$


LEMMA 7. If $p \in \mathbf{P}, p \Vdash$ " $x: \omega \rightarrow 2$ is in $M_{2}-M_{1}$ ", and $\alpha<\omega_{1}$, then there are $\bar{p} \leqslant p$ and $z: \omega \rightarrow 2$ in $M$ so that $\bar{p} \Vdash$ “ $\operatorname{deg}(G(\alpha)) \leqslant \operatorname{deg}(x) \vee \operatorname{deg}(z) "$.

Proof. Choose $\overline{\bar{p}} \leqslant p$ so that $\alpha \in \operatorname{supp}(\overline{\bar{p}})$ and $\left\langle p_{s} \mid s \in \operatorname{Seq}\right\rangle$ with associated $\left\langle\sigma_{s}\right| s \in$ Seq $\rangle$ to be a P-fusion sequence for $x$ below $\overline{\bar{p}}$. Let $\bar{p}=p_{\omega}=$ $M_{n \in \omega} W_{\ln (s)=n} p_{s}$ be the fusion of that sequence. Then

$$
\bar{p} \Vdash “ G(\alpha)=\bigcap\left\{p_{s}(\alpha) \mid \sigma_{s}=x \uparrow \ln \left(\sigma_{s}\right)\right\} " .
$$

Let $z$ be a real in $M$ which recursively codes $\left\{\left\langle p_{s}(\alpha), \sigma_{s}\right\rangle \mid s \in\right.$ Seq $\} . z$ provides a reduction procedure which can be used to compute $G(\alpha)$ from $x$.

Proposition. Let $M, \kappa$ be as in the hypothesis of Theorem 2, $G=\langle G(\alpha) \mid \alpha<\kappa\rangle$ be P-generic over $M$. Then $M[G]$ is a cardinal-preserving extension of $M$ in which $2^{\omega}=\kappa$, and there is an $X$ which has cardinality $\omega_{1}$ and is a maximal independent set of Turing degrees.

Proof. Standard results on forcing show that forcing with $\mathbf{P}$ preserves cardinalities and cofinalities, $M_{1} \vDash$ " $2^{\omega}=\omega_{1}$ " and $M_{2} \Vdash$ " $2^{\omega}=\kappa$ ".

The $G(\alpha)$ for $\alpha<\omega_{1}$ are mutually generic so their Turing degrees form an independent set. Let $X$ be a maximal independent set of Turing degrees in $M_{1}$ containing $\left\{\operatorname{deg}(G(\alpha)) \mid \alpha<\omega_{1}\right\}$. (In fact, by another fusion argument, $X=$ $\left\{\operatorname{deg}(G(\alpha)) \mid \alpha<\omega_{1}\right\}$, but this is not crucial here.) $\overline{\bar{X}}=\omega_{1}$ since $X \subset M_{1}$.

$X$ remains maximal in $M_{2}$, for suppose $x$ is a real in $M_{2}-M_{1}$. By Lemma 7 , for some real $z \in M, \operatorname{deg}(G(0)) \leqslant \operatorname{deg}(x) \vee \operatorname{deg}(z)$. By Lemma 6 , there is an $\alpha<\omega_{1}$ so that $\alpha \neq 0, \operatorname{deg}(z) \leqslant \operatorname{deg}(G(\alpha))$. Thus $\operatorname{deg}(G(0)) \leqslant \operatorname{deg}(x) \vee \operatorname{deg}(G(\alpha))$, showing that $\operatorname{deg}(x)$ cannot be added to $X$ without destroying independence.

This proves Theorem 2.

Further remarks; open questions. As was previously mentioned, Sacks conjectured in [9] that: If $A$ is a locally countable partial order of size at most continuum, then $A \rightarrow \mathscr{D}$. His results show that the conjecture follows from $\mathrm{CH}$; the same methods extend to show that Martin's Axiom (MA) also implies the conjecture. Proving the conjecture from ZFC remains an open problem. Theorem 2 places a limitation on possible methods of proof since it implies that an embedding of a size $\omega_{1}$ suborder of $A$ may not extend to an embedding of $A$.

Theorem 1 shows that it is not always the case that all locally countable upper semilattices of size at most continuum are embeddable in $\mathscr{D}$. We conjecture (with some confidence) that this is the case under MA. A further question: assuming MA, can any such upper semilattice be embedded as an initial segment of $\mathscr{D}$ ?

\section{REFERENCES}

1. J. Baumgartner and R. Laver, Iterated perfect set forcing, Ann. Math. Logic 17 (1979), 271-288.

2. S. C. Kleene and E. L. Post, The upper semi-lattice of degrees of recursive unsolvability, Ann. of Math. (2) 59 (1954), 379-407.

3. P. Cohen, Set theory and the continuum hypothesis, Benjamin, New York, 1966.

4. A. H. Lachlan, Distributive initial segments of the degrees of unsolvability, Math. Logik Grundlag. Math. 14 (1968), 457-472.

5. A. H. Lachlan and R. Lebeuf, Countable initial segments of the degrees of unsolvability, J. Symbolic Logic 41 (1976), 289-300. 
6. M. Lerman, Initial segments of the degrees of unsolvability, Ann. of Math. (2) 93 (1971), 365-389.

7. J. M. Rubin, The existence of an $\omega_{1}$ initial segment of the Turing degrees, Notices Amer. Math. Soc. 26 (1979), Abstract \#79T-A168, p. A-425.

8. Distributive uncountable initial segments of the degrees of unsolvability, Notices Amer. Math. Soc. 26 (1979), Abstract \#79T-E74, p. A-619.

9. G. E. Sacks, Degrees of unsolvability, Ann. of Math. Studies, no. 55, Princeton Univ. Press, Princeton, N.J., 1963.

10. Pure Math., vol. 13, Amer. Math. Soc., Providence, R.I., 1971, pp. 331-355.

11. S. G. Simpson, Degrees of unsolvability: a survey of results, Handbook of Mathematical Logic (J. Barwise, editor), North-Holland, Amsterdam, 1977.

Department of Mathematics, Massachusetts Institute of Technology, Cambridge, MasSACHUSETTS 02139

Department of Mathematics, University of Chicago, Chicago, Illinois 60637 